\title{
The generalized Stieltjes transform and its inverse
}

John H. Schwarz

California Institute of Technology, Pasadena, California 91125

(Received 3 June 2004; accepted 16 September 2004; published online 23 December 2004)

The generalized Stieltjes transform (GST) is an integral transform that depends on a parameter $\rho>0$. In previous work a convenient form of the inverse transformation was derived for the case $\rho=3 / 2$. This paper generalizes that result to all $\rho$ $>0$. It is a well-known fact that the GST can be formulated as an iterated Laplace transform, and that therefore its inverse can be expressed as an iterated inverse Laplace transform. The form of the inverse transform derived here is a onedimensional integral that is considerably simpler. (C) 2005 American Institute of Physics. [DOI: 10.1063/1.1825077]

\section{INTRODUCTION}

In Ref. 1 we encountered the integral equation

$$
g(\zeta)=\int_{0}^{\infty}\left(\zeta^{2}+\mu^{2}\right)^{-3 / 2} f(\mu) \mathrm{d} \mu, \quad|\arg \zeta|<\pi / 2
$$

where $g(\zeta)$ was a known function, and we needed to solve for $f(\mu)$. That paper proved that if $g$ has suitable analyticity and asymptotic properties, which were satisfied for the specific function of interest, then

$$
f(\mu)=-\frac{i \mu^{2}}{\pi} \int_{0}^{\pi} \cos \theta g(-i \mu \cos \theta) \mathrm{d} \theta .
$$

This result is reviewed in Ref. 2 .

By a change of variables, Eq. (2) can be rewritten in the form

$$
f(\mu)=\frac{\mu}{\pi i} \int_{-i \mu}^{i \mu} \frac{\zeta}{\sqrt{\mu^{2}+\zeta^{2}}} g(\zeta) \mathrm{d} \zeta .
$$

Then letting $y=\mu^{2}$ and $z=\zeta^{2}$, as well as $F(y)=(1 / 2 \mu) f(\mu)$ and $G(z)=g(\zeta)$, we obtain

$$
G(z)=\int_{0}^{\infty}(y+z)^{-3 / 2} F(y) \mathrm{d} y, \quad|\arg z|<\pi
$$

and

$$
F(y)=\frac{1}{4 \pi i} \int_{\mathcal{C}_{y}} \frac{G(z)}{\sqrt{y+z}} \mathrm{~d} z .
$$

The contour $\mathcal{C}_{y}$ starts and ends at $-y$ and encloses the origin in the counterclockwise sense.

It is convenient to have a $y$-independent contour, so letting $z=w y$ we obtain 


$$
F(y)=\frac{1}{4 \pi i} \sqrt{y} \int_{\mathcal{C}} \frac{G(y w)}{\sqrt{1+w}} \mathrm{~d} w,
$$

where $\mathcal{C}$ is a contour that starts and ends at the point $w=-1$ enclosing the origin in the counterclockwise sense. It could be chosen to be the unit circle, for instance. Note that it is not really a closed contour, since $G(z)$ has a branch cut running along the negative real axis. An integration by parts allows this to be rewritten in the form

$$
F(y)=-\frac{1}{2 \pi i} y^{3 / 2} \int_{\mathcal{C}} \sqrt{1+w} G^{\prime}(y w) \mathrm{d} w .
$$

In this paper, we will formulate and prove a one-parameter generalization of the preceding result. Specifically, we claim that the integral transform

$$
G(z)=\int_{0}^{\infty}(y+z)^{-\rho} F(y) \mathrm{d} y, \quad|\arg z|<\pi
$$

has as its inverse transform

$$
F(y)=-\frac{1}{2 \pi i} y^{\rho} \int_{\mathcal{C}}(1+w)^{\rho-1} G^{\prime}(y w) \mathrm{d} w .
$$

This integral converges for $\rho>0$. The special case discussed above corresponds to $\rho=3 / 2$. The counterpart of Eq. (6), obtained by integration by parts, namely

$$
F(y)=\frac{1}{2 \pi i}(\rho-1) y^{\rho-1} \int_{\mathcal{C}}(1+w)^{\rho-2} G(y w) \mathrm{d} w,
$$

is less general, since it is only valid for $\rho>1$. This is allowed in the $\rho=3 / 2$ case, of course.

We should note for the record what is being assumed about $F$ and $G$. Specifically, $F(y)$, which is only defined on the positive real axis, is allowed to be an arbitrary distribution (or "generalized function"). We require that there exists a number $\alpha$ with $0<\alpha<\rho$ such that $\left|\int_{y_{1}}^{y_{2}} y^{\alpha-\rho} F(y) \mathrm{d} y\right|$ is bounded by a number independent of $y_{1}$ and $y_{2}$ for all $0<y_{1}<y_{2}$. The function $G(z)$ is then holomorphic throughout the cut plane $|\arg z|<\pi$, and there exists a positive real number $\beta$ such that $\left|z^{\beta} G(z)\right|$ is bounded at infinity.

The $\rho=1$ case of Eq. (8), known as the Stieltjes transform, is discussed in Widder's classic treatise on the Laplace transform. ${ }^{3}$ The only other cases considered by Widder are positive integer values of $\rho$, which are related to the $\rho=1$ case by differentiation. Following Ref. 4 , we refer to the case of arbitrary $\rho$ as the generalized Stieltjes transform (GST). (In Ref. 5 it is called a Stieltjes transform of index $\rho$.) The formula for the inverse GST in Eq. (9) does not seem to have been found previously. Certainly, it does not appear in Refs. 3-5 or 6.

Much of the literature on the GST is concerned with the asymptotic behavior of $G(z)$ for large $|z|{ }^{7}$ We will not address that topic here. As it happens, in Ref. 1 we were interested in deducing the asymptotic behavior of $F$ associated with a given $G$.

In the special case $\rho=1$ the transform in Eq. (8) reduces to the Stieltjes transform

$$
G(z)=S_{z}[F]=\int_{0}^{\infty} \frac{F(y)}{y+z} \mathrm{~d} y .
$$

Since the Stieltjes transform is well understood, this case provides an instructive test of the proposed inverse transform. Setting $\rho=1$ in Eq. (9) gives an expression that can be integrated explicitly to give 


$$
F(y)=\lim _{\epsilon \rightarrow 0^{+}} \frac{1}{2 \pi i}(G(-y-i \epsilon)-G(-y+i \epsilon)) .
$$

Given the stated analytic and asymptotic properties of $G(z)$, it is a simple consequence of Cauchy's theorem that this is the correct solution of Eq. (11) for $y>0$.

In order to convince oneself that Eqs. (8) and (9) are plausible for all $\rho>0$, it is instructive to consider a simple example. Specifically, if one chooses

$$
F(y)=y^{\nu-1},
$$

then the integral in Eq. (8) converges for $0<\nu<\rho$ and gives

$$
G(z)=z^{\nu-\rho} B(\nu, \rho-\nu),
$$

where $B(u, v)$ is the Euler beta function. It is straightforward to verify that this pair of functions also satisfies Eq. (9).

\section{OTHER VERSIONS OF THE INVERSE TRANSFORM}

The change of variables $z=w y$ allows us to rewrite Eq. (9) in the alternative form

$$
F(y)=-\frac{1}{2 \pi i} \int_{\mathcal{C}_{y}}(y+z)^{\rho-1} G^{\prime}(z) \mathrm{d} z .
$$

As before, the contour $\mathcal{C}_{y}$ starts and ends at the point $z=-y$, encircling the origin in the counterclockwise sense. If $\rho>1$, an integration by parts brings Eq. (15) to the form

$$
F(y)=\frac{1}{2 \pi i}(\rho-1) \int_{\mathcal{C}_{y}}(y+z)^{\rho-2} G(z) \mathrm{d} z .
$$

Since this is only well-defined for $\rho>1$, Eqs. (9) and (15) are more general than Eq. (16).

Let us define the quantity that appears on the right-hand side of Eq. (12) to be

$$
\Delta(t)=\frac{1}{2 \pi i} \lim _{\epsilon \rightarrow 0^{+}}(G(-t-i \epsilon)-G(-t+i \epsilon)), \quad t>0 .
$$

We showed that $F(y)=\Delta(y)$ when $\rho=1$, but this is not the case for other values of $\rho$. By shrinking the contour $\mathcal{C}_{y}$ down to the cut, Eq. (16) takes the form

$$
F(y)=(\rho-1) \int_{0}^{y}(y-t)^{\rho-2} \Delta(t) \mathrm{d} t .
$$

In similar fashion, Eq. (15) gives rise to

$$
F(y)=\int_{0}^{y}(y-t)^{\rho-1} \Delta^{\prime}(t) \mathrm{d} t .
$$

However, these formulas are only correct if the behavior of $G(t)$ near the origin is such that these integrals exist. The contour integral versions of these formulas are more general, since they do not have this restriction.

Equations (18) and (19) have the structure of Abel transforms. The inverse Abel transform is well known and can be used to give a formula for the discontinuity across the cut, $\Delta(t)$, in terms of the original (generalized) function $F(y)$. A version that is suitable if $\rho<2$ and $F(0)=0$ is 


$$
\Delta(t)=\frac{\sin \pi \rho}{\pi(1-\rho)} \int_{0}^{t}(t-y)^{1-\rho} F^{\prime}(y) \mathrm{d} y .
$$

This gives the known result for $\rho=1$, namely $\Delta(t)=F(t)$. It can be checked for the simple example $F(y)=y^{\nu-1}$ discussed earlier.

\section{PROOF OF THE MAIN RESULT}

Let us review how the GST is related to the Laplace transform, for which we use the following notation:

$$
\mathcal{L}_{x}[F]=\int_{0}^{\infty} e^{-x y} F(y) \mathrm{d} y .
$$

Inserting the identity

$$
(y+z)^{-\rho}=\frac{1}{\Gamma(\rho)} \int_{0}^{\infty} x^{\rho-1} e^{-x(y+z)} \mathrm{d} x
$$

into Eq. (8) gives

$$
G(z)=\frac{1}{\Gamma(\rho)} \int_{0}^{\infty} x^{\rho-1} e^{-x z} \mathcal{L}_{x}[F] \mathrm{d} x=\frac{1}{\Gamma(\rho)} \mathcal{L}_{z}\left[x^{\rho-1} \mathcal{L}_{x}[F]\right] .
$$

This recasts Eq. (8) as a Laplace transform of a Laplace transform. In particular, setting $\rho=1$, this gives the well-known result that the Stieltjes transform is the square (in the operator sense) of the Laplace transform, i.e., $\mathcal{S}=\mathcal{L}^{2}$.

Equation (23) implies that

$$
\mathcal{L}_{x}[F]=\Gamma(\rho) x^{1-\rho} \mathcal{L}_{x}^{-1}[G],
$$

where $\mathcal{L}^{-1}$ denotes an inverse Laplace transform. A second inverse Laplace transform gives the formal inversion of Eq. (23),

$$
F(y)=\Gamma(\rho) \mathcal{L}_{y}^{-1}\left[x^{1-\rho} \mathcal{L}_{x}^{-1}[G]\right] .
$$

This can be made very explicit by using the standard contour integral realization of the inverse Laplace transformation. ${ }^{5}$ The new claim is that Eq. (25) can be simplified to take the form of Eq. (9).

Let us now carry out some similar manipulations of Eq. (9). Substituting

$$
G^{\prime}(y w)=-\int_{0}^{\infty} \mathrm{d} t e^{-t y w} t \mathcal{L}_{t}^{-1}[G]
$$

into Eq. (9) and taking the Laplace transform of both sides recasts Eq. (9) in the form

$$
\mathcal{L}_{x}[F]=\Gamma(\rho+1) \frac{1}{2 \pi i} \int_{\mathcal{C}} \mathrm{d} w(1+w)^{\rho-1} \int_{0}^{\infty} \mathrm{d} t(x+t w)^{-\rho-1} t \mathcal{L}_{t}^{-1}[G] .
$$

Comparing Eqs. (24) and (27), we see that their equivalence requires the identity

$$
\frac{t \rho}{2 \pi i} \int_{\mathcal{C}} \mathrm{d} w(1+w)^{\rho-1}(x+t w)^{-\rho-1}=x^{-1-\rho} \delta(x-t),
$$

or equivalently 
(a)

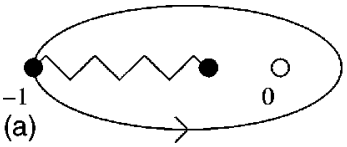

(b)

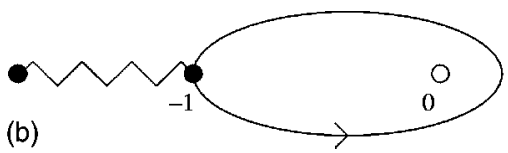

(c)

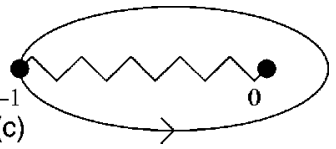

FIG. 1. The contour $\mathcal{C}$ starts and ends at the point $w=-1$ enclosing the origin in the counterclockwise sense. Three possible branch cut configurations are depicted.

$$
\frac{\rho}{2 \pi i} \int_{\mathcal{C}} \mathrm{d} w(1+w)^{\rho-1}(x+t w)^{-\rho-1}=x^{-\rho} \delta(x-t) .
$$

Another way of understanding the necessity of Eq. (29) is to consider the special case $F(y)$ $=\delta(y-t)$. In this case the GST is $G(z)=(t+z)^{-\rho}$. The inverse transform Eq. (9) for this choice of $G(z)$ corresponds precisely to Eq. (29). This remarkable equation, which is required to hold for $x$, $t>0$, is the heart of the matter. Its proof, which is rather nontrivial, is presented in the next section.

\section{REPRESENTATION OF A DELTA FUNCTION}

This section proves the key formula, namely Eq. (29). Consider the left-hand side of Eq. (29),

$$
\chi_{\rho}(x, t)=\frac{\rho}{2 \pi i} \int_{\mathcal{C}} \mathrm{d} w(1+w)^{\rho-1}(x+t w)^{-\rho-1},
$$

where $x, t>0$ and $\mathcal{C}$ is the contour shown in Fig. 1. By making the change of variables $w \rightarrow 1 / w$, it is easy to prove that $\chi_{\rho}(x, t)=\chi_{\rho}(t, x)$. For $t>x$ the singularity structure is shown in Fig. 1(a), and the contour can be pushed off to infinity, giving zero for the integral. For $t<x$, the contour encloses no singularity [see Fig. 1(b)], so the result is again zero, as required by $x \leftrightarrow t$ symmetry.

Since $\chi_{\rho}(x, t)$ vanishes for $t \neq x$, it must be some sort of distribution concentrated at $t=x$. If we assume that it is proportional to a delta function, i.e., $f(x) \delta(x-t)$, it is easy to derive $f(x)$. Integrating over $t$ from 0 to $y$, gives

$$
f(x) \theta(y-x)=\frac{1}{2 \pi i} \int_{\mathcal{C}} \frac{\mathrm{d} w}{w}(1+w)^{\rho-1}\left[x^{-\rho}-(x+y w)^{-\rho}\right] .
$$

The first term on the right-hand side is an elementary contour integral and gives $x^{-\rho}$. The second term is also easily evaluated by Cauchy's theorem. It gives $-x^{-\rho}$ if $x>y$ and zero if $y>x$. Thus we deduce that $f(x)=x^{-\rho}$, and hence

$$
\chi_{\rho}(x, t)=x^{-\rho} \delta(x-t)=t^{-\rho} \delta(x-t) .
$$

Equation (32) is the correct result, but the derivation given above is not rigorous. It assumes that the distribution is proportional to a delta function, and that it does not involve any derivatives of delta functions. A more careful analysis that is sensitive to such terms if they are present involves checking the proposed answer by integrating both sides against the test function $e^{-t y}$, i.e., comparing the Laplace transform of both sides of the equation. Thus we need to show that

$$
\int_{0}^{\infty} \mathrm{d} t \chi_{\rho}(x, t) e^{-t y}=x^{-\rho} e^{-x y}
$$

Evaluating the Laplace transform of Eq. (30) gives (after some simple manipulations)

$$
\int_{0}^{\infty} \mathrm{d} t \chi_{\rho}(x, t) e^{-t y}=\frac{\rho}{2 \pi i} y^{\rho} \int_{\mathcal{C}} \mathrm{d} w(1+w)^{\rho-1} I(x y w),
$$

where 


$$
I(u)=e^{u} \int_{u}^{\infty} e^{-v} v^{-\rho-1} \mathrm{~d} v .
$$

In order to re-express this function in a more convenient form, we first note that

$$
I^{\prime}(u)=I(u)-u^{-\rho-1} .
$$

Let us now assume that $\rho$ is not an integer, which is the case of most interest. This equation is then solved by an expression of the form

$$
I(u)=I_{1}(u)+u^{-\rho} I_{2}(u),
$$

where $I_{1}(u)$ and $I_{2}(u)$ are regular at $u=0$. The first term is $I_{1}(u)=\Gamma(-\rho) e^{u}$. Its contribution to Eq. (34) is zero, since there is no singularity inside the contour. The function that matters is $I_{2}$, which satisfies the differential equation

$$
u I_{2}^{\prime}(u)-(u+\rho) I_{2}(u)+1=0 .
$$

Substituting a power series expansion,

$$
I_{2}(u)=\sum_{n=0}^{\infty} c_{n} u^{n}
$$

one obtains the recursion relation $(n+1-\rho) c_{n+1}=c_{n}$. Thus, since $c_{0}=I_{2}(0)=1 / \rho$, we conclude that

$$
c_{n}=\frac{\Gamma(1-\rho)}{\rho \Gamma(n+1-\rho)} .
$$

To determine the contribution of each term in Eq. (39) to Eq. (34), we need to evaluate

$$
\frac{1}{2 \pi i} \int_{\mathcal{C}} \mathrm{d} w(1+w)^{\rho-1} w^{n-\rho}=-\frac{\sin \pi(n-\rho)}{\pi} B(n-\rho+1, \rho)=\frac{(-1)^{n}}{\rho c_{n} n !} .
$$

Combining these results, the $c_{n}$ factors cancel, and we learn that

$$
\int_{0}^{\infty} \mathrm{d} t \chi_{\rho}(x, t) e^{-t y}=x^{-\rho} \sum_{n=0}^{\infty} \frac{(-x y)^{n}}{n !}=x^{-\rho} e^{-x y},
$$

which is the result we set out to prove. Even though this derivation needs to be modified when $\rho$ is a positive integer, the result clearly is valid in that case as well. This completes the proof of Eq. (33) and hence of the inverse transform Eq. (9).

The derivation of Eq. (32) (and hence of the inverse GST) can be simplified considerably by using the alternative representation of the inversion formula presented in Eq. (19) together with well-known properties of hypergeometric functions. Specifically, using

$$
I\left(z, \rho, \rho^{\prime}\right)=\int_{-1}^{0} \mathrm{~d} w(1+w)^{\rho-1}(1+z w)^{-p^{\prime}}=\frac{1}{\rho}(1-z)^{\rho-\rho^{\prime}}{ }_{2} F_{1}\left(\rho, \rho-\rho^{\prime}+1 ; \rho+1 ; z\right)
$$

one can show that

$$
\begin{aligned}
\chi_{\rho}(x, t) & =\frac{1}{2 \pi i} \rho x^{-\rho-1} \lim _{\epsilon \rightarrow 0+}\left[I\left(\rho, \rho+1, \frac{t}{x}+i \epsilon\right)-I\left(\rho, \rho+1, \frac{t}{x}-i \epsilon\right)\right]=\frac{1}{\pi} x^{-\rho} \lim \frac{\epsilon t}{(x-t)^{2}+(\epsilon t)^{2}} \\
& =x^{-\rho} \delta(x-t) .
\end{aligned}
$$

The restriction on the behavior of $G(z)$ at the origin mentioned previously can be circumvented by introducing a modified function $G_{\delta}(z)=G(z+\delta), \delta>0$, and letting $\delta \rightarrow 0$ in the final result. The 
advantage of this approach is that it is applicable to a wider class of distributions on the real line, namely those that can be written as the difference of the limiting values on the real axis from above and below of two functions, holomorphic in the upper and lower half-plane, respectively. ${ }^{8,9}$

\section{AN ALTERNATIVE DERIVATION}

In this section we present a simpler, though less general, derivation of the inverse transform. It follows from Cauchy's theorem and the required analytic and asymptotic properties of $G(z)$ that

$$
G(z)=\int_{0}^{\infty} \frac{\Delta(t)}{z+t} \mathrm{~d} t,
$$

where $\Delta(t)$ is defined in Eq. (17). The validity of this formula requires that $\Delta(t)$ is not too singular as $t \rightarrow 0$, so that the integral exists. This is a significant restriction, since if $F \sim y^{\nu-1}$ with $\nu>0$ for small $y$, then $G(z) \sim z^{\nu-\rho}$ for small $z$. Thus one would need that $\rho<\nu+1$. No such assumption has been made previously, which is why the derivation in this section is less general.

Equation (45) is precisely a Stieltjes transform, $G=\mathcal{S}[\Delta]$. We noted earlier that this is an iterated Laplace transform, $\mathcal{S}=\mathcal{L}^{2}$. Therefore,

$$
\mathcal{L}_{x}^{-1}[G]=\mathcal{L}_{x}[\Delta]
$$

Comparing this with Eq. (24), which we obtained from Eq. (8), we learn that

$$
\mathcal{L}_{x}[F]=\Gamma(\rho) x^{1-\rho} \mathcal{L}_{x}[\Delta]
$$

Now using $\mathcal{L}_{x}\left[t^{\nu-1}\right]=\Gamma(\nu) x^{-\nu}$, this becomes

$$
\mathcal{L}_{x}[F]=(\rho-1) \mathcal{L}_{x}\left[t^{\rho-2}\right] \mathcal{L}_{x}[\Delta]
$$

By the convolution theorem, this implies that

$$
F(y)=(\rho-1) \int_{0}^{y}(y-t)^{\rho-2} \Delta(t) \mathrm{d} t,
$$

which is Eq. (18). The alternative form in Eq. (19) extends the range of validity for $\rho$, but is even more restricted in its requirements for the behavior of $\Delta(t)$ at the origin. As we pointed out in Sec. II, there is no such issue for the corresponding contour integrals.

\section{CONCLUSION}

In this paper we considered the generalized Stieltjes transform

$$
G(z)=\int_{0}^{\infty}(y+z)^{-\rho} F(y) \mathrm{d} y, \quad|\arg z|<\pi
$$

for $\rho>0$ and proved that it has as its inverse transform

$$
F(y)=-\frac{1}{2 \pi i} y^{\rho} \int_{\mathcal{C}}(1+w)^{\rho-1} G^{\prime}(y w) \mathrm{d} w .
$$

The integration contour is described in the text.

\section{ACKNOWLEDGMENTS}

The author is grateful to Y. H. He, M. Spradlin, and A. Volovich for their collaboration in Ref. 1 , which contains the $\rho=3 / 2$ case of the result described here. This work was supported in part by the U.S. Department of Energy under Grant No. DE-FG03-92-ER40701. 
${ }^{1}$ Y. H. He, J. H. Schwarz, M. Spradlin, and A. Volovich, Phys. Rev. D 67, 086005 (2003).

${ }^{2}$ J. H. Schwarz, hep-th/0312283, to appear in a memorial volume for Ian Kogan.

${ }^{3}$ D. V. Widder, The Laplace Tansform (Princeton University Press, Princeton, NJ, 1946).

${ }^{4}$ A. Erdelyi, Tables of Integral Transforms (McGraw-Hill, New York, 1954), Vol. 2.

${ }^{5}$ A. I. Zayed, Handbook of Function and Generalized Function Transformations (CRC Press, New York, 1996).

${ }^{6}$ O. P. Misra and J. L. Lavoine, Transform Analysis of Generalized Functions (North-Holland, Amsterdam, 1986).

${ }^{7}$ P. D. Tuan and D. Elliott, Math. Comput. 26, 213 (1972); J. P. McClure and R. Wong, J. Inst. Math. Appl. 22, 129 (1978); R. Wong, J. Math. Anal. Appl. 72, 740 (1979); J. L. Lopez, and C. Ferreira, Stud. Appl. Math. 108, 187 (2002).

${ }^{8}$ H. J. Bremermann, Distributions, Complex Variables, and Fourier Transforms (Addison-Wesley, Reading, MA, 1965).

${ }^{9}$ H. G. Tillmann, Math. Z. 77, 106 (1961). 